PROCEEDINGS OF THE

AMERICAN MATHEMATICAL SOCIETY

Volume 130, Number 2, Pages 311-314

S 0002-9939(01)06083-X

Article electronically published on May 25, 2001

\title{
WEDDERBURN'S FACTORIZATION THEOREM APPLICATION TO REDUCED $K$-THEORY
}

\author{
R. HAZRAT
}

(Communicated by Lance W. Small)

\begin{abstract}
This article provides a short and elementary proof of the key theorem of reduced $K$-theory, namely Platonov's Congruence theorem. Our proof is based on Wedderburn's factorization theorem.
\end{abstract}

Let $D$ be a division algebra with center $F$. If $a \in D$ is algebraic over $F$ of degree $m$, then by Wedderburn's factorization theorem one can find $m$ conjugates of $a$ such that the sum and the product of them are in $F$. This observation has been used in many different circumstances to give a short proof of known theorems of central simple algebras. (See 8] for a list of these theorems.) Here we will use this fact to prove Platonov's congruence theorem.

The non-triviality of the reduced Whitehead group $S K_{1}(D)$ was first shown by V. P. Platonov who developed a so-called reduced $K$-theory to compute $S K_{1}(D)$ for certain division algebras. The key step in his theory is the "congruence theorem" which is used to connect $S K_{1}(\bar{D})$, where $\bar{D}$ is a residue division algebra of $D$ to $S K_{1}(D)$. This in effect enables one to compute the group $S K_{1}(D)$ for certain division algebras. (See [5] and [6].)

Before we describe the congruence theorem, we employ Wedderburn's factorization theorem to obtain a result regarding normal subgroups of division algebras.

Suppose that $D$ has index $n$. Let $N$ be a normal subgroup of the group of units $D^{*}$ of $D$. Let $a \in N$ with the minimal polynomial $f(x) \in F[x]$ of degree $m$. Then from the theory of central simple algebras we have the following equality:

$$
f(x)^{n / m}=x^{n}-\operatorname{Trd}_{D / F}(a) x^{n-1}+\cdots+(-1)^{n} N r d_{D / F}(a),
$$

where $N r d_{D / F}: D^{*} \rightarrow F^{*}$ is the reduced norm, $\operatorname{Trd}_{D / F}: D \rightarrow F$ is the reduced trace and the right-hand side of the equality is the reduced characteristic polynomial of $a$. (See [7], §9.)

Using Wedderburn's factorization theorem for the minimal polynomial $f(x)$ of $a$, one obtains $f(x)=\left(x-d_{1} a d_{1}^{-1}\right) \cdots\left(x-d_{m} a d_{m}^{-1}\right)$ for certain $d_{i} \in D$. Now from the equality (1), it follows that $\operatorname{Nrd}_{D / F}(a)$ is the product of $n$ conjugates of $a$. Since $N$ is a normal subgroup of $D^{*}$, it follows that $\operatorname{Nrd}_{D / F}(a) \in N$. Therefore $\left.N r d\right|_{N}: N \longrightarrow Z(N)$ is well defined, where $Z(N)=F^{*} \cap N$ is the center of the group $N$.

Received by the editors April 13, 2000 and, in revised form, June 12, 2000.

2000 Mathematics Subject Classification. Primary 16A39.

Key words and phrases. Division algebra, reduced $K$-theory, congruence theorem, reduced Whitehead group. 
Before stating the main lemma, we fix some notation.

Let $\mu_{n}(F)$ denote the group of $n$-th roots of unity in $F$ and let $Z\left(D^{\prime}\right)$ denote the center of the commutator subgroup $D^{\prime}$ of $D^{*}$. Let $D^{(1)}$ stand for the kernel of the reduced norm. Observe that $\mu_{n}(F)=F^{*} \cap D^{(1)}$ and $Z\left(D^{\prime}\right)=F^{*} \cap D^{\prime}$. If $G$ is a group, denote by $G^{n}$ the subgroup of $G$ generated by all $n$-th powers of elements of $G$. If $H$ and $K$ are subgroups of $G$, denote by $[H, K]$ the subgroup of $G$ generated by all mixed-commutators $[h, k]=h k h^{-1} k^{-1}$, where $h \in H$ and $k \in K$.

We are now in a position to state our main lemma which is interesting in its own right.

Lemma 1. Let $D$ be a division algebra with center $F$, of index $n$. Let $N$ be a normal subgroup of $D^{*}$. Then $N^{n} \subseteq\left(F^{*} \cap N\right)\left[D^{*}, N\right]$.

Proof. Let $a \in N$. As stated above, using Wedderburn's factorization theorem, $\operatorname{Nrd}_{D / F}(a)=d_{1} a d_{1}{ }^{-1} \cdots d_{n} a d_{n}{ }^{-1}$. But

$$
d_{1} a d_{1}{ }^{-1} \cdots d_{n} a d_{n}{ }^{-1}=\left[d_{1}, a\right] a\left[d_{2}, a\right] a \cdots\left[d_{n}, a\right] a=a^{n} d_{a}
$$

for some $d_{a} \in\left[D^{*}, N\right]$. This implies that $a^{n}=N r d_{D / F}(a) d_{a}{ }^{-1}$. Therefore $N^{n} \subseteq$ $\left(F^{*} \cap N\right)\left[D^{*}, N\right]$.

Let $N=D^{*}$. Then by the above Lemma, for any $x \in D^{*}, x^{n}=N r d_{D / F}(x) d_{x}$ where $d_{x} \in D^{\prime}$. This shows that the group $G(D)=D^{*} / F^{*} D^{\prime}$ is a torsion group of bounded exponent $n$. Some algebraic properties of this group are studied in [4].

In order to describe Platonov's congruence theorem, we need to recall some concepts from valued division algebras. Let $D$ be a finite dimensional division algebra with center a Henselian field $F$. Recall that a valuation $v$ on a field $F$ is called Henselian if and only if $v$ has a unique extension to each field algebraic over $F$. Therefore $v$ has a unique extension also denoted by $v$ to $D(11)$. Denote by $V_{D}, V_{F}$ the valuation rings of $v$ on $D$ and $F$, respectively and let $M_{D}, M_{F}$ denote their maximal ideals and $\bar{D}, \bar{F}$ their residue division algebra and residue field, respectively. We let $\Gamma_{D}, \Gamma_{F}$ denote the value groups of $v$ on $D$ and $F$, respectively, and $U_{D}, U_{F}$ the groups of units of $V_{D}, V_{F}$, respectively. Furthermore, we assume that $D$ is a tame division algebra, i.e., $C h a r \bar{F}$ does not divide $i(D)$, the index of $D$.

Platonov's congruence theorem asserts that if $D$ is a tame division algebra over a Henselian field $F$, then $\left(1+M_{D}\right) \cap D^{(1)} \subseteq D^{\prime}$. This is the crucial theorem of reduced $K$-theory which is proved in [5] (note that [5] provides a lengthy and complicated proof for the special case of a complete discrete valuation of rank 1, and 3 . notes that the same proof works for the general case of tame Henselian valued division algebras). Here we give a short and elementary proof of this fact.

Theorem 2 (Congruence Theorem). Let $D$ be a tame division algebra over a Henselian field $F=Z(D)$, of index $n$. Then $\left(1+M_{D}\right) \cap D^{(1)}=\left[D^{*}, 1+M_{D}\right]$.

Proof. First we show that $\left(1+M_{F}\right) \cap D^{(1)}=1$. Let $1+f \in 1+M_{F}$. If $1+f \in D^{(1)}$, then $(1+f)^{n}=1$. But $v\left((1+f)^{n}-1\right)=v(f)$. This shows that $f=0$ and so our claim. Now take $N=1+M_{D}$. By Lemma 1 ,

$$
\left(1+M_{D}\right)^{n} \subseteq\left(\left(1+M_{D}\right) \cap F^{*}\right)\left[D^{*},\left(1+M_{D}\right)\right] .
$$


Since the valuation is tame and Henselian, Hensel's lemma shows that $\left(1+M_{D}\right)^{n}=$ $1+M_{D}$. Therefore $1+M_{D}=\left(1+M_{F}\right)\left[D^{*},\left(1+M_{D}\right)\right]$. Now using the fact that $\left(1+M_{F}\right) \cap D^{(1)}=1$, the theorem follows.

Remark. There is an elegant proof of the congruence theorem by A. Suslin in [9], in the case of a discrete valuation of rank 1. This proof uses substantial results from valuation theory and the fact that the group $S K_{1}(D)$ is torsion of bounded exponent $n=i(D)$. Using results of Ershov in [3], Suslin's proof can be written for arbitrary tame Henselian division algebras.

Having the congruence theorem, it is easy to see, in the case of discrete valuation of rank 1 , that the sequence

$$
S K_{1}(\bar{D}) \rightarrow S K_{1}(D) \rightarrow L_{1} / L_{\sigma^{-1}} \rightarrow 1
$$

is exact, where $L=\operatorname{Nrd}(\bar{D}), L_{1}=L \cap N_{Z(\bar{D}) / \bar{F}}^{-1}(1)$ and $L_{\sigma^{-1}}=$ the image of $L$ under the homomorphism $a \mapsto \sigma(a) a^{-1}$, where $\langle\sigma\rangle=\operatorname{Gal}(Z(\bar{D}) / \bar{F})$. This leads to computations of $S K_{1}(D)$ for certain division algebras. (See [5], 6] and [9].)

Another look at the proof of Theorem 2 shows that $1+M_{D} \subseteq\left(1+M_{F}\right) D^{\prime}$ and therefore $1+M_{D} \subseteq U_{F} D^{\prime}$. Put $G(\bar{D})=\bar{D}^{*} / \bar{F}^{*} \overline{D^{\prime}}$. In many applications, it is easy to obtain information about the residue data of division algebras. The following theorem gives an explicit formula for the group $S K_{1}(D)$ when the group $G(\bar{D})$ is trivial.

Theorem 3. Let $D$ be a tame division algebra over a Henselian field $F=Z(D)$, of index $n$. If $G(\bar{D})=1$, then $S K_{1}(D)=\mu_{n}(F) / Z\left(D^{\prime}\right)$.

Proof. The reduction map $U_{D} \longrightarrow \bar{D}^{*}$ induces an isomorphism $\bar{D}^{*} \longrightarrow U_{D} / 1+M_{D}$, $\bar{a} \mapsto\left(1+M_{D}\right) a$. Since $1+M_{D} \subseteq U_{F} D^{\prime}$, it follows that

$$
\bar{D}^{*} / \bar{F}^{*} \overline{D^{\prime}} \stackrel{\simeq}{\longrightarrow} U_{D} / U_{F} D^{\prime} \text {. }
$$

Now if $G(\bar{D})=\bar{D}^{*} / \bar{F}^{*} \overline{D^{\prime}}=1$, then $U_{D}=U_{F} D^{\prime}$. But $D^{(1)} \subseteq U_{D}$. This shows that $D^{(1)}=\mu_{n}(F) D^{\prime}$. Using the fact that $\mu_{n}(F) \cap D^{\prime}=Z\left(D^{\prime}\right)$, the theorem follows.

Note that Hensel's lemma implies that $\mu_{n}(F) \simeq \mu_{n}(\bar{F})$. In particular if $D$ is a totally ramified division algebra, i.e., $\bar{D}=\bar{F}$, then $G(\bar{D})=1$.

Example 4. Let $\mathbb{C}$ be the field of complex numbers and $r$ be a nonnegative integer. Let $D_{1}=\mathbb{C}\left(\left(x_{1}\right)\right)$ and define $\sigma_{1}: D_{1} \rightarrow D_{1}$ by the rule $\sigma_{1}\left(x_{1}\right)=-x_{1}$. Now let $D_{2}=D_{1}\left(\left(x_{2}, \sigma_{1}\right)\right)$ and set $D_{3}=D_{2}\left(\left(x_{3}\right)\right)$. Again define $\sigma_{3}: D_{3} \rightarrow D_{3}$ by $\sigma_{3}\left(x_{3}\right)=-x_{3}$. In general, if $i$ is even, set $D_{i+1}=D_{i}\left(\left(x_{i+1}\right)\right)$ and if $i$ is odd, define $\sigma_{i}: D_{i} \rightarrow D_{i}$ by $\sigma_{i}\left(x_{i}\right)=-x_{i}$ and $D_{i+1}=D_{i}\left(\left(x_{i+1}, \sigma_{i}\right)\right)$. By Hilbert's construction (see [1], $\S 1$ and $\S 24), D=D_{2 r}=\mathbb{C}\left(\left(x_{1}, \cdots, x_{2 r}, \sigma_{1}, \cdots, \sigma_{2 r-1}\right)\right)$ is a division algebra with center $F=\mathbb{C}\left(\left(x_{1}^{2}, x_{2}^{2}, \cdots, x_{2 r-1}^{2}, x_{2 r}^{2}\right)\right)$ and $n=i(D)=2^{r}$. Finally define $v: D^{*} \rightarrow \Gamma_{D}=Z^{2 r}$ by the rule $v\left(\sum c_{i} x_{1}^{i_{1}} \cdots x_{2 r}^{i_{2 r}}\right)=\left(i_{1}, \cdots, i_{2 r}\right)$ where $i_{1}, \cdots, i_{2 r}$ are the smallest powers of the $x_{i}$ 's in the lexicographic order. It can be observed that $v$ is a tame valuation and $\bar{D}=\mathbb{C}$ and $\bar{F}=\mathbb{C}$. Therefore $G(\bar{D})=1$. Theorem 3 implies that $S K_{1}(D)=\mu_{n}(F) / Z\left(D^{\prime}\right)$. From the multiplication rule in $D$, it follows that

$$
D^{\prime} \subseteq\left\{ \pm 1+\sum_{i>0} c_{i} x_{1}^{i_{1}} \cdots x_{2 r}^{i_{2 r}}\right\}
$$


Since $Z\left(D^{\prime}\right) \subseteq \mu_{n}(F)$, it follows that $Z\left(D^{\prime}\right)=\{1,-1\}$. But $\mu_{n}(F)=\mu_{n}(\bar{F})=\mathbb{Z}_{2^{r}}$; hence $S K_{1}(D)=\mathbb{Z}_{2^{r-1}}$.

In [4], as another application of Lemma 1 , we obtain theorems of reduced $K$ theory which previously required heavy machinery, as a simple consequence of this approach.

\section{ACKNOWLEDGMENTS}

I wish to thank Anthony Bak who read the note and made numerous corrections.

\section{REFERENCES}

1. P. Draxl, Skew Field, London Math. Soc. Lecture Note Ser. Vol 81, Cambridge, Univ. Press. Cambridge, 1983. MR 85a:16022

2. P. Draxl, M. Kneser (eds.), $S K_{1}$ von Schiefkörpern, Lecture Notes in Math. Vol 778, Springer, Berlin, 1980. MR 82b:16014

3. Y. Ershov, Henselian valuation of division rings and the group $S K_{1}(D)$, Math USSR Sb. 45 (1983), 63-71.

4. R. Hazrat, $S K_{1}$-like functors for division algebras, J. of Algebra, to appear.

5. V. P. Platonov, The Tannaka-Artin problem and reduced K-theory, Math USSR Izv. 10 (1976), 211-243.

6. V. P. Platonov, V. Yanchevskii, Algebra IX, Finite dimensional division algebras, Encyclopaedia Math. Sci. 77, Springer, Berlin, 1995.

7. I. Reiner, Maximal Orders, Academic Press, London, 1975. MR 52:13910

8. L. Rowen, Y. Segev, The multiplicative group of a division algebra of degree 5 and Wedderburn's factorization theorem, Contemp. Math., 259, Amer. Math. Soc., 2000. CMP 2001:01

9. A. Suslin, $S K_{1}$ of division algebras and Galois cohomology, Advances in Soviet Math 4, Amer. Math. Soc. (1991), 75-99.

10. J.-P. Tignol, A. R. Wadsworth, Totally ramified valuations on finite-dimensional division algebras, Tran. Amer. Math. Soc. 302 (1) (1987), 223-250. MR 88j:16025

11. A. R. Wadsworth, Extending valuations to finite dimensional division algebras, Proc. Amer. Math. Soc. 98 (1986), 20-22. MR 87i:16025

Department of Mathematics, University of Bielefeld, P. O. Box 100131, 33501 BieleFELD, Germany

E-mail address: rhazrat@mathematik.uni-bielefeld.de 